Research Article

\title{
Simultaneous Quantification of Six Bioactive Components in Decoction of Ziziphi spinosae Semen Using Ultrahigh Performance Liquid Chromatography Coupled with Triple-Quadrupole Mass Spectrometry
}

\author{
Xiao Liu $\mathbb{D}^{1},{ }^{1}$ Xiaochai Zhu, ${ }^{1}$ Hui Zhu, ${ }^{1}$ Li Xie, ${ }^{1}$ Jia Ma, ${ }^{2}$ Yinghui Xu, ${ }^{2}$ Qigang Zhou, \\ Zejun $W u,{ }^{1}$ and Baochang Cai $\mathbb{D}^{1}$ \\ ${ }^{1}$ School of Pharmacy, Nanjing University of Chinese Medicine, Nanjing, China \\ ${ }^{2}$ Fushun Central Hospital, Fushun, China \\ ${ }^{3}$ Department of Pharmacology, Pharmacy College, Nanjing Medical University, Nanjing, China
}

Correspondence should be addressed to Xiao Liu; liuxiao04_0@163.com and Baochang Cai; bccai@126.com

Received 25 May 2018; Accepted 5 September 2018; Published 1 November 2018

Academic Editor: Federica Pellati

Copyright ( 2018 Xiao Liu et al. This is an open access article distributed under the Creative Commons Attribution License, which permits unrestricted use, distribution, and reproduction in any medium, provided the original work is properly cited.

\begin{abstract}
This paper was conducted to develop a method containing ultrahigh performance liquid chromatography coupled with triplequadrupole tandem mass spectrometry for simultaneous quantification of six bioactive components in the decoction of Ziziphi spinosae Semen. Analysis was performed on an Agilent ZORBAX Extend- $\mathrm{C}_{18}$ column $(2.1 \times 100 \mathrm{~mm}, 1.8 \mu \mathrm{m})$ and eluted with a mobile phase system consisting of acetonitrile and water under a gradient program with a flow rate of $0.3 \mathrm{ml} / \mathrm{min}$. The injection volume was $2 \mu \mathrm{l}$. Multiple-reaction monitoring scanning detection was employed for quantification with an electrospray ion source in the negative ion mode. All the six compounds showed good linearities $(r \geq 0.9996)$. The LODs of the six bioactive compounds were $0.039 \mathrm{ng} / \mathrm{ml}, 0.092 \mathrm{ng} / \mathrm{ml}, 3.112 \mathrm{ng} / \mathrm{ml}, 2.131 \mathrm{ng} / \mathrm{ml}, 0.099 \mathrm{ng} / \mathrm{ml}$, and $0.071 \mathrm{ng} / \mathrm{ml}$ for spinosin, $6^{\prime \prime \prime}$-feruloylspinosin, jujuboside A, jujuboside B, camelliaside B, and betulinic acid, respectively. The LOQs were $0.118 \mathrm{ng} / \mathrm{ml}$, $0.276 \mathrm{ng} / \mathrm{ml}, 9.336 \mathrm{ng} / \mathrm{ml}, 6.393 \mathrm{ng} / \mathrm{ml}, 0.299 \mathrm{ng} / \mathrm{ml}$, and $0.213 \mathrm{ng} / \mathrm{ml}$ for spinosin, $6^{\prime \prime \prime}$-feruloylspinosin, jujuboside A, jujuboside B, camelliaside B, and betulinic acid, respectively. According to our knowledge, it was the first time to establish a method with high efficiency and accuracy for the quantification of six bioactive components in the decoction of Ziziphi spinosae Semen, which would provide references for quality control and evaluation of Ziziphi spinosae Semen.
\end{abstract}

\section{Introduction}

Ziziphi spinosae Semen (ZSS; suanzaoren in Chinese), the seeds of Ziziphus jujuba Mill. var. spinosa (Bunge) Hu ex H. F. Chou, has been used as a typical sedative-hypnotic traditional Chinese medicine (TCM) in clinic for thousands of years in China $[1,2]$. There are many different categories of chemical constituents in ZSS, including flavonoids, saponins, and triterpenoids. Spinosin, jujuboside A, and jujuboside $\mathrm{B}$ were reported to be active components which exerted sedative-hypnotic effect [3-8]. It was found that the sedative-hypnotic effect of ZSS might be due to the action of its chemical constitutions, such as spinosin, jujuboside A, and jujuboside $B$, on serotonergic system and GABA receptors [9-11]. Several studies have reported the analysis of relative chemical constituents in ZSS; however, the limitation of the performance coming from traditional detectors, such as UV and ELSD, made it quite difficult to detect a variety of complex components in ZSS quickly and accurately $[12,13]$. Recently, tandem mass spectrometry with high sensitivity and selectivity has become the mainstream detection method due to its advantages in simultaneous quantitative and qualitative analyses. The combination of tandem mass spectrometry with UHPLC made it possible for the effective and reliable detection of complex constitutions in TCM, which would compensate for the 3 shortcomings in 
the previous research studies [14-17]. In fact, chemical constituents of ZSS have been studied in some previous research studies $[18,19]$, but none of them could determine the contents of its active components efficiently due to the diversity of these constituents.

Decoction was the most common and traditional using form during TCM clinical application [20]. However, organic solvents were used for sample extraction in most of the existing research studies [21], which was different from TCM actual application in clinics. And this might lead to a limitation of these research studies for guiding ZSS clinical application and quality control.

\section{Experimental}

2.1. Materials and Reagents. Methanol (LC-MS grade) and acetonitrile (LC-MS grade) were obtained from E. Merck (Merck, Darmstadt, Germany). Ultrapurified water $(18.25 \Omega)$ was daily prepared with a Milli-Q water purification system (Millipore Corporation, Bedford, MA, USA). All the other regents were of analytical purity and commercially available.

Reference standards including spinosin (batch no. S-043150626), 6 6"'-feruloylspinosin (batch no. A-011-170731), jujuboside A (batch no. S-045-160809), and jujuboside B (batch no. S-046-151205) were purchased from Chengdu Ruifensi Biological Technology Co. Ltd. Camelliaside B (batch no. 170313-080) and betulinic acid (batch no. JYB 201701) were purchased from JinYiBai Biological Technology Co. Ltd. The purity of these reference standards was all above $98.0 \%$.

Ten different batches of ZSS samples collected from Shandong (batch no. 1-5), Shanxi (batch no. 6-7), and Hebei (batch no. 8-10) provinces, which are the main production regions of ZSS in China, were used as experimental materials after a careful quality evaluation according to the Chinese Pharmacopoeia 2015 edition-part one [1].

2.2. Preparation of Standard Solutions. The stock resolutions of 6 components were independently prepared. Then, the mixed reference standard solution was obtained by moderate dilution of the stock solution with methanol. The concentrations of the 6 components in the final mixed reference standards solution used for identification and quantification were $6.312,5.885,0.991,0.668,0.640$, and $4.504 \mu \mathrm{g} / \mathrm{ml}$. A series of reference standard solutions with six different concentrations were prepared from the final mixed reference standard solution by diluting $1,2,4,8,16$, and 32 times, respectively. Finally, the concentration ranges of each reference standard in the series of reference standard solutions were $0.197-6.312 \mu \mathrm{g} / \mathrm{ml}, \quad 0.184-5.885 \mu \mathrm{g} / \mathrm{ml}$, $0.031-0.991 \mu \mathrm{g} / \mathrm{ml}, \quad 0.021-0.668 \mu \mathrm{g} / \mathrm{ml}, \quad 0.020-0.640 \mu \mathrm{g} / \mathrm{ml}$, and $0.141-4.504 \mu \mathrm{g} / \mathrm{ml}$, respectively.

2.3. Sample Preparation. According to the experience of clinical application of ZSS, $10 \mathrm{~g}$ dried powder of ZSS (60 mesh sieve passed) was accurately weighed and decocted twice with boiling water $(1: 10, w / v$ and $1: 8, w / v$, resp.), each for $1 \mathrm{~h}$. Solutions were filtered with 4-layer meshes and merged together. Water was added to the merged decoction to fix a final volume of $200 \mathrm{ml}$ to obtain the decoction of ZSS. $0.2 \mathrm{ml}$ of the decoction sample was transferred into a $5 \mathrm{ml}$ volumetric flask, and then appropriate methanol was added to the fixed volume and mixed. Appropriate amount of the mixture was removed and centrifuged at $12000 \mathrm{r} / \mathrm{min}$ for $5 \mathrm{~min}$. The supernatant liquor was taken out, and $2 \mu \mathrm{l}$ of which was finally injected into the UHPLC-QQQ-MS/MS system for analysis.

2.4. UHPLC-QQQ-MS/MS Conditions. A Shimazu 30A UHPLC (Shimadzu, Japan) equipped with a binary solvent system was used to perform chromatographic separation. An Agilent $\mathrm{C}_{18}$ column $(2.1 \mathrm{~mm} \times 100 \mathrm{~mm}, 1.8 \mu \mathrm{m})$ was used at $25^{\circ} \mathrm{C}$. A linear gradient program was performed with the mobile phases consisting of eluent A (water) and eluent $\mathrm{B}$ (acetonitrile) as follows: $0-1.2 \mathrm{~min}, 5-31 \% \mathrm{~B} ; 1.2-$ $2.5 \mathrm{~min}, 31-95 \% \mathrm{~B} ; 2.5-4.5 \mathrm{~min}, 95-100 \% \mathrm{~B}$; and $4.5-6 \mathrm{~min}$, $100-100 \% \mathrm{~B}$. The flow rate was $0.3 \mathrm{ml} / \mathrm{min}$.

An MS/MS system (Triple Quad 5500, AB Sciex, USA) with an electrospray ionization source (ESI) was used to perform analysis of the six bioactive components in decoction of ZSS. The detection was performed under negative ion mode with an MRM detection way. The acquisition parameters of mass spectrometry are listed as follows: atomization gas $\left(\mathrm{N}_{2}\right)$ of 55 psi; auxiliary heating gas of 55 psi; curtain gas of 35 psi; turbospray temperature of $550^{\circ} \mathrm{C}$; and ion spray voltage floating of $-4500 \mathrm{~V}$. The MRM transitions were tuned to the optimal for meeting the standard of the experiment. Also, declustering potential and collision energy parameters of the six components were debugged to optimal in order to meet the purpose of accurate detection of all the components.

2.5. Quantification Analysis. AB Sciex MultiQuant 2.1 Software was applied to perform the quantification of the six components. Peak area ratios were calculated and plotted against concentrations by using the software.

\section{Results and Discussion}

3.1. Optimization of the Chromatographic and Spectrometric Conditions. It was reported in some previous studies that the six components analyzed in this study were active components which exerted sedative-hypnotic effect [3-8]. Using the UHPLC-QQQ-MS/MS method developed in this experiment, the six components with different types of structures could be well separated and quantified within 6 minutes. Methanol or acetonitrile as the organic phases and water or $0.1 \%$ formic acid in water $(\mathrm{v}: \mathrm{v})$ as the aqueous phases were tested to obtain good chromatographic peaks. In order to accurately determine the six components, the ion fragments of MRM transition of these analytes were detected. All the chemical information of these components was further confirmed by comparisons of retention time to the mixed reference standard. The detailed UHPLC-ESIMS/MS detection parameters of the six components are shown in Table 1 . The representative $\mathrm{MS}^{2}$ spectrum and 
TABLE 1: HPLC-ESI-MS/MS detection parameters of the six components.

\begin{tabular}{|c|c|c|c|c|c|c|c|}
\hline Analyte & Mass data & $t_{\mathrm{R}}(\min )$ & Ion mode & $\mathrm{MS}^{1}(m / z)$ & $\mathrm{MS}^{2}(m / z)$ & $\mathrm{DP} / \mathrm{V}$ & $\mathrm{CE} / \mathrm{eV}$ \\
\hline Spinosin & 608.1741 & 2.40 & $\mathrm{ESI}^{-}$ & 607.10 & 427.10 & -26.46 & -41.60 \\
\hline $6^{\prime \prime \prime \prime}$-Feruloylspinosin & 784.2215 & 2.54 & $\mathrm{ESI}^{-}$ & 783.40 & 427.20 & -25.83 & -50.92 \\
\hline Jujuboside A & 1207.3533 & 2.75 & $\mathrm{ESI}^{-}$ & 1206.60 & 1073.30 & -60.00 & -60.00 \\
\hline Jujuboside B & 1044.5505 & 2.83 & $\mathrm{ESI}^{-}$ & 1043.60 & 911.40 & -27.20 & -49.77 \\
\hline Camelliaside B & 726.2007 & 2.36 & $\mathrm{ESI}^{-}$ & 725.30 & 284.00 & -23.72 & -53.07 \\
\hline Betulinic acid & 456.3604 & 5.16 & $\mathrm{ESI}^{-}$ & 455.30 & 455.30 & -97.00 & -20.00 \\
\hline
\end{tabular}

MRM chromatograms of six analytes in this experiment are shown in Figure 1.

\subsection{Assay Validation}

3.2.1. Linearity and Range. AB Sciex MultiQuant 2.1 software was used to determine regression lines as well as to ensure precision. The mixed reference standard solution was diluted to a series of standard solutions with different concentrations in accordance with gradients of 1, 2, 4, 8, 16, and 32 times, which could be used to make the standard curves. At the same time, the reference standard solution was gradually diluted and detected, and the limitation of quantitation (LOQ) and limitation of detection (LOD) were performed. LOQ was determined as the concentration whose $S / N$ was 10 , and LOD was determined as the concentration whose $S / N$ was 3 . All the $r$ values were above 0.9990 in this experiment, which indicated that the linear relationship of this method for each analyte was good. The regression equations and linear ranges of all the detected components are shown in Table 2.

3.2.2. Precision Test. Proper amount of mixed reference standard solution was taken and detected for six times under the testing conditions. The peak areas of the six components were recorded, which could be used to investigate the precision by calculating the RSDs. The results showed that the RSDs of the six components were $0.60 \%, 2.51 \%, 2.75 \%$, $2.91 \%, 1.12 \%$, and $2.62 \%$. The precision of this method was excellent.

3.2.3. Repeatability Test. Six copies of ZSS powder, taken from the same batch, each for $10 \mathrm{~g}$, were used to prepare the trial solution in accordance with the method in Section 2.3. Peak areas of the six components were recorded to investigate the repeatability of the method by calculating RSDs. The results showed that RSDs were $1.20 \%, 2.64 \%$, $2.60 \%, 2.21 \%, 2.98 \%$, and $1.18 \%$, indicating that the repeatability of this method met the requirement.

3.2.4. Stability Test. The trial solution was taken from the samples prepared in Section 2.3 randomly and tested at 0,2, $4,8,12,24$, and $48 \mathrm{~h}$ under the analytical conditions. The peak areas of the six components were recorded to calculate the RSDs, which were used to investigate the stability of the samples. The result that the RSDs of the six components were $2.96 \%, 2.53 \%, 2.82 \%, 2.10 \%, 2.31 \%$, and $2.72 \%$ indicated that the stability of the samples was good.

3.2.5. Recovery Test. Dried powder of ZSS ( $5 \mathrm{~g}, 60$ mesh sieve passed) was accurately weighed, and fair level of reference standard solution was added, which would be then prepared as the trial solution according to Section 2.3. Determination was performed under the analytical conditions, and the peak areas of the six components were recorded in order to calculate RSDs and recoveries. The data shown in Table 3 indicated that the RSDs and recoveries for six analytes were eligible.

3.2.6. Quantification of the Six Components in ZSS Samples. Ten different batches of ZSS samples collected from their main producing regions in China were prepared as the trial solution in accordance with the method in Section 2.3. Determination of the components was performed under the analytical conditions given in Section 2.4, and the peak areas which would be used to calculate the mass fraction were recorded. The contents of the six bioactive constituents of the decoctions of ZSS samples from three different producing regions were in the ranges of 1375.17-2612.84, 297.27-788.05, 60.20-112.75, 47.34-114.60, 10.17-46.13, and $141.78-616.83 \mu \mathrm{g} / \mathrm{g}$ (Table 4). Interestingly, among all these six components determined, the contents of spinosin and jujuboside $\mathrm{A}$, the most important active constituents which exerted sedative-hypnotic effects, were relatively stable in the ten batches of ZSS samples from three different regions. As for the other four components, their contents fluctuated more clearly in these samples as the regions changed. Taking a comprehensive evaluation into consideration, the contents of the six bioactive ingredients in the samples from Hebei province (samples with the No. from 8 to 10) were the highest, followed by the samples from Shandong province (samples with the No. from 1 to 5) being the second one. The results of this experiment indicated that the contents of bioactive components were quite different in ZSS from different regions. Therefore, the origin might be a key factor that should be considered when ZSS was clinically applied for its sedative-hypnotic action.

\section{Conclusions}

A rapid and accurate method for determination of six bioactive components in the decoction of ZSS was established for the first time in this paper. Besides accurate and quick quantitation, the mass information and ion fragments 


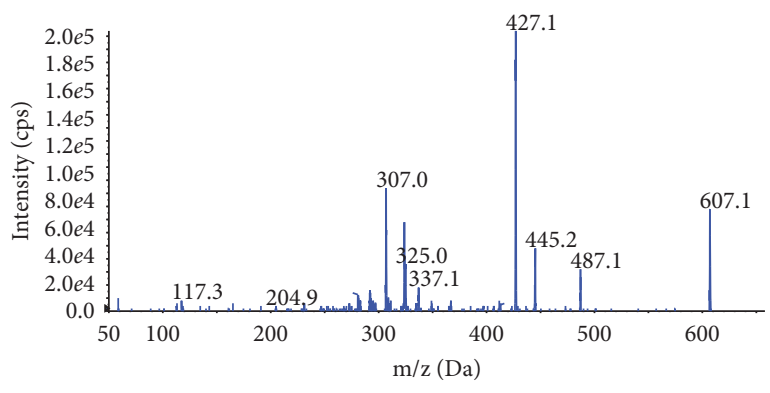

(a)

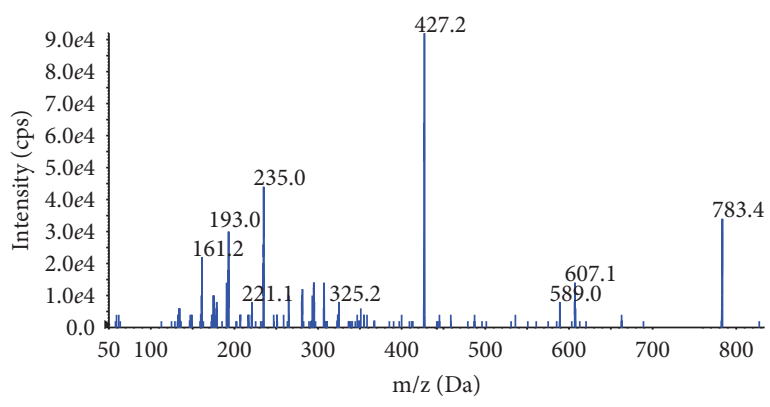

(c)

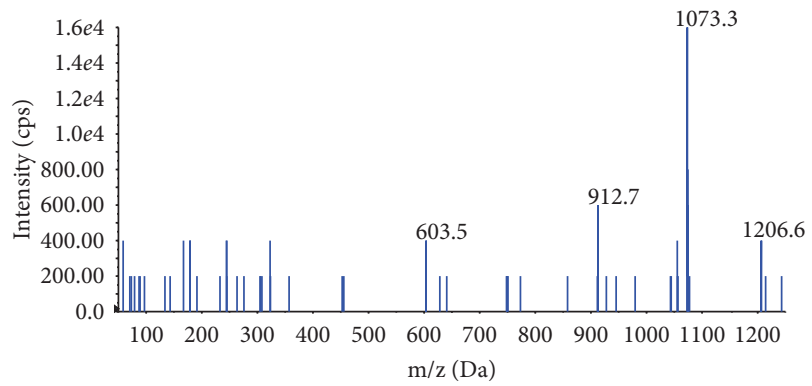

(e)

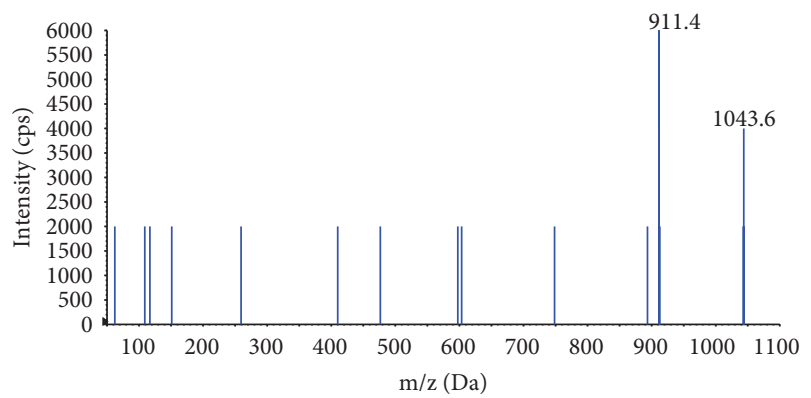

(g)

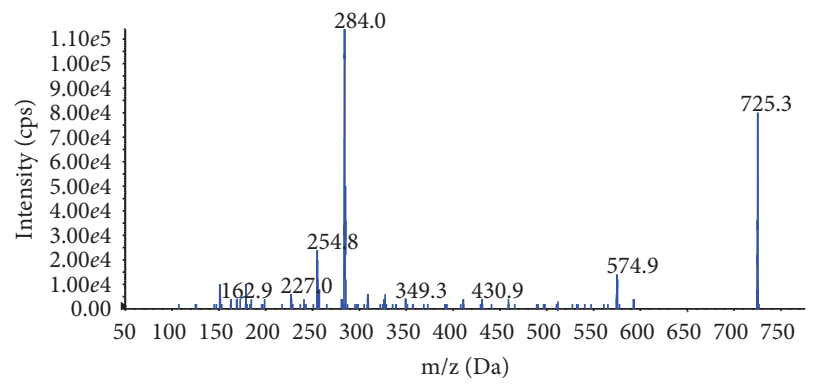

(i)

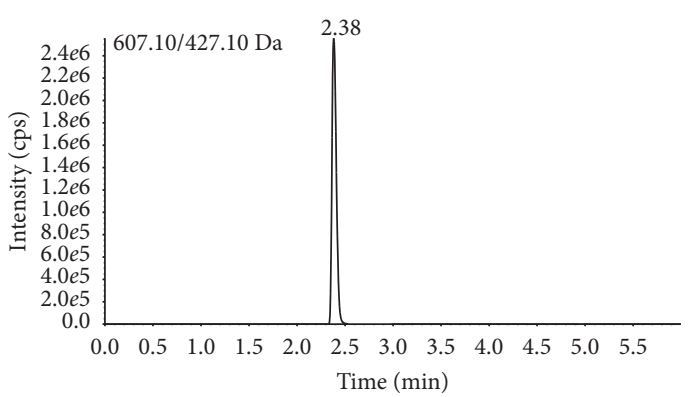

(b)

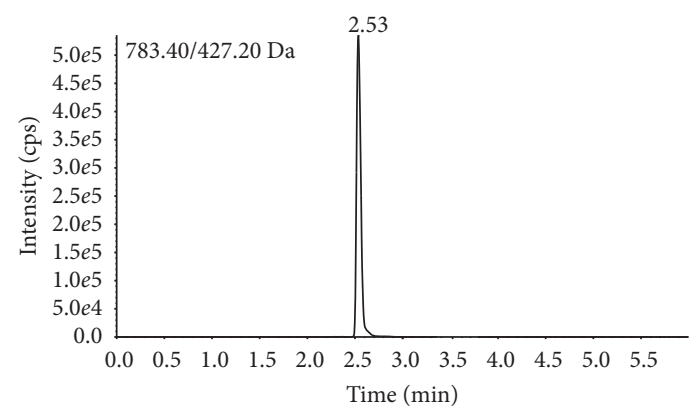

(d)

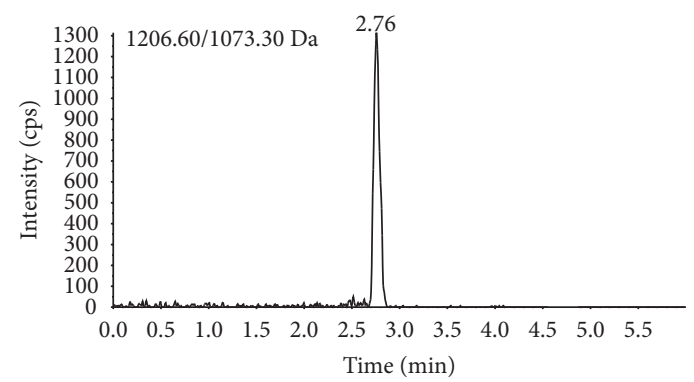

(f)

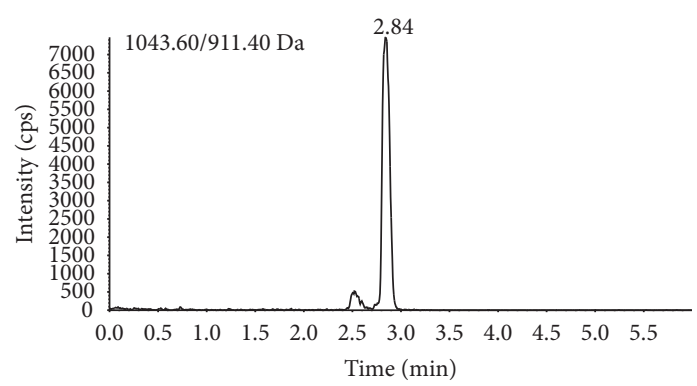

(h)

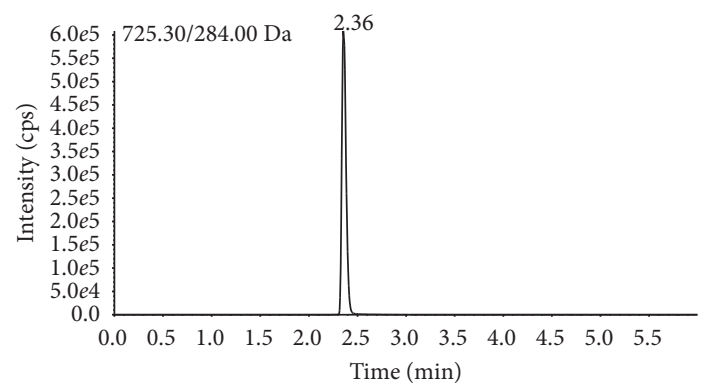

(j)

Figure 1: Continued. 


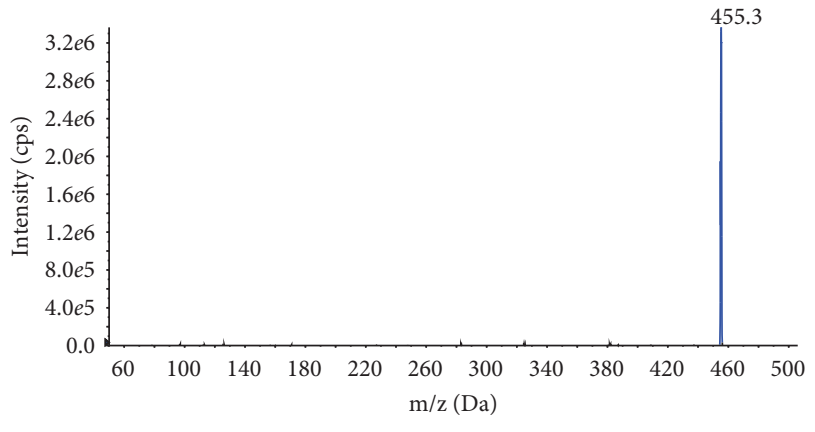

(k)

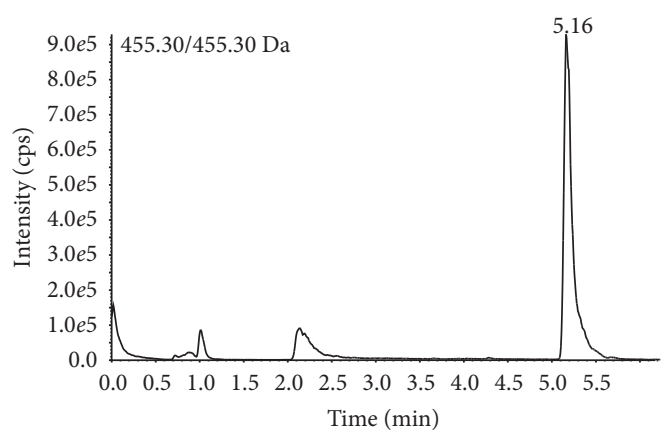

(l)

Figure 1: The representative MS ${ }^{2}$ spectrum of (a) spinosin; (c) 6"' - feruloylspinosin; (e) jujuboside A; (g) jujuboside B; (i) camelliaside B; and (k) betulinic acid, and MRM chromatograms of (b) spinosin; (d) 6"' -feruloylspinosin; (f) jujuboside A; (h) jujuboside B; (i) camelliaside $B$; and (l) betulinic acid of six analytes in reference standards under experimental conditions.

TABLE 2: Calibration curves, correlation coefficients $(r)$, and linear ranges of the six components.

\begin{tabular}{lccccc}
\hline Analyte & Calibration curve & $r$ & Linear range $(\mu \mathrm{g} / \mathrm{ml})$ & LODs $(\mathrm{ng} / \mathrm{ml})$ & LOQs $(\mathrm{ng} / \mathrm{ml})$ \\
\hline Spinosin & $y=2.08 e^{6} x+7.35 e^{5}$ & 0.9996 & $0.197-6.312$ & 0.039 & 0.118 \\
$6^{\prime \prime \prime}$-Feruloylspinosin & $y=1.32 e^{6} x+3.79 e^{5}$ & 0.9997 & $0.184-5.885$ & 0.092 & 0.276 \\
Jujuboside A & $y=1.05 e^{4} x-241.13$ & 0.9996 & $0.031-0.991$ & 2.112 & 9.336 \\
Jujuboside B & $y=7.28 e^{4} x-2926.02$ & 0.9996 & $0.021-0.668$ & $0.020-0.640$ & 0.099 \\
Camelliaside B & $y=1.10 e^{7} x+6.01 e^{5}$ & 0.9997 & $0.141-4.504$ & 0.071 & 0.299 \\
Betulinic acid & $y=1.00 e^{7} x+9.11 e^{5}$ & 0.9998 & & & 0.213 \\
\hline
\end{tabular}

TABLE 3: Recovery data of the six analytes $(n=6)$.

\begin{tabular}{|c|c|c|c|c|c|}
\hline Analyte & Sample content $(\mu \mathrm{g})$ & Actual amount of control $(\mu \mathrm{g})$ & Amount of control found $(\mu \mathrm{g})$ & Recovery (\%) & RSD (\%) \\
\hline Spinosin & 15.25 & $15.58 \pm 0.0 .26$ & $15.60 \pm 0.19$ & $100.14 \pm 1.00$ & 0.97 \\
\hline $6^{\prime \prime \prime \prime}$-Feruloylspinosin & 7.50 & $7.34 \pm 0.25$ & $7.33 \pm 0.24$ & $99.88 \pm 0.02$ & 1.81 \\
\hline Jujuboside A & 1.32 & $1.33 \pm 0.19$ & $1.35 \pm 0.16$ & $98.21 \pm 0.03$ & 2.64 \\
\hline Jujuboside B & 0.88 & $0.84 \pm 0.06$ & $0.84 \pm 0.05$ & $99.90 \pm 0.03$ & 2.88 \\
\hline Camelliaside B & 0.79 & $0.81 \pm 0.02$ & $0.81 \pm 0.03$ & $99.81 \pm 0.03$ & 2.97 \\
\hline Betulinic acid & 2.90 & $2.86 \pm 0.05$ & $2.88 \pm 0.05$ & $100.70 \pm 0.01$ & 1.31 \\
\hline
\end{tabular}

TABLE 4: The contents of the 6 components in the decoction of ZSS samples ( $\mu \mathrm{g} / \mathrm{g})$.

\begin{tabular}{|c|c|c|c|c|c|c|}
\hline No. & Spinosin & $6^{\prime \prime \prime}$-Feruloylspinosin & Jujuboside A & Jujuboside B & Camelliaside B & Betulinic acid \\
\hline 1 & 2069.00 & 783.50 & 105.75 & 80.40 & 58.92 & 403.40 \\
\hline 2 & 1774.83 & 576.17 & 83.05 & 67.97 & 30.41 & 598.50 \\
\hline 3 & 2063.67 & 622.50 & 78.52 & 80.62 & 37.89 & 141.78 \\
\hline 4 & 1375.17 & 297.27 & 60.20 & 47.77 & 10.17 & 440.30 \\
\hline 5 & 1507.17 & 300.08 & 57.80 & 61.18 & 13.19 & 616.83 \\
\hline 6 & 1748.33 & 436.28 & 66.70 & 50.51 & 25.60 & 469.35 \\
\hline 7 & 1520.83 & 304.38 & 67.37 & 80.17 & 12.54 & 177.92 \\
\hline 8 & 2123.97 & 598.51 & 88.95 & 47.34 & 31.88 & 293.07 \\
\hline 9 & 2209.67 & 639.33 & 95.22 & 90.38 & 36.33 & 228.03 \\
\hline 10 & 2612.84 & 788.05 & 112.75 & 114.60 & 46.13 & 187.72 \\
\hline Average & 1900.55 & 534.61 & 81.63 & 72.09 & 30.31 & 355.69 \\
\hline $\mathrm{SD}$ & 363.56 & 180.19 & 18.04 & 20.38 & 14.84 & 165.92 \\
\hline
\end{tabular}

of MRM transition could provide meaningful reference for the identification of the analytes. It was hoped that the analytical method developed would provide a reference for the quality control and evaluation of ZSS. At the same time, the results that great differences existed in the contents of the six bioactive components in the samples from different regions indicated that the origin factor might be related to ZSS clinical efficacy. 


\section{Abbreviations}

UHPLC-

Ultrahigh performance liquid

QQQ-MS:

chromatography method coupled with

triple-quadrupole mass spectrometry

ZSS:

TCM:

Ziziphi spinosae Semen

UV:

Traditional Chinese medicine

Ultraviolet

ELSD:

Evaporative light-scattering detector

MRM:

\section{Data Availability}

The data used to support the findings of this study are included within the article.

\section{Conflicts of Interest}

The authors declare that there are no conflicts of interests.

\section{Acknowledgments}

This work was financially supported by the National Natural Science Foundation of China (Nos. 81573603 and 81503207), Major Projects of the Natural Science Foundation of Jiangsu Higher Education Institutions (No. 17KJA360006), and Jiangsu Province "333" Project.

\section{References}

[1] The Pharmacopoeia Commission of People's Republic of China, Pharmacopoeia of the People's Republic of China. (2015 ed), vol. 1, pp. 366-367, China Medical Pharmaceutical Science and Technology Publishing House, Beijing, China, 2015.

[2] M. C. Zhang, Y. Q. Zhang, and J. B. Xie, "Simultaneous determination of jujuboside A, B and betulinic acid in semen Ziziphi spinosae by high performance liquid chromatographyevaporative light scattering detection," Journal of Pharmaceutical and Biomedical Analysis, vol. 48, no. 5, pp. 1467-1470, 2008.

[3] W. Kim, T. B. Zhao, H. Y. Zhang et al., "Quantitative and pattern recognition analyses of magnoflorine, spinosin, $6^{\prime \prime \prime}$ feruloylspinosin and jujuboside A by HPLC in Zizyphi Semen," Archives of Pharmacal Research, vol. 37, no. 9, pp. 1139-1147, 2014.

[4] J. X. Cao, Q. Y. Zhang, S. Y. Cui et al., "Hypnotic effect of jujubosides from Semen Ziziphi spinosae," Journal of Ethnopharmacology, vol. 130, no. 1, pp. 163-166, 2010.

[5] X. S. Fang, J. F. Hao, H. Y. Zhou et al., "Pharmacological studies on the sedative-hypnotic effect of Semen Ziziphi spinosae (Suanzaoren) and Radix et Rhizoma Salviae miltiorrhizae (Danshen) extracts and the synergistic effect of their combinations," Phytomedicine, vol. 17, no. 1, pp. 75-80, 2010.

[6] Y. Yan, Q. Li, C. H. Du et al., "Investigation of the potentially effective components of Semen Ziziphi spinosae based on "in vitro to vivo" translation approach," Acta Pharmaceutica Sinica, vol. 52, pp. 283-290, 2017.

[7] I. H. Jung, H. E. Lee, S. J. Park et al., "Ameliorating effect of spinosin, a C-glycoside flavonoid, on scopolamine-induced memory impairment in mice," Pharmacology Biochemistry and Behavior, vol. 120, pp. 88-94, 2014.

[8] L. E. Wang, X. Y. Cui, S. Y. Cui et al., "Potentiating effect of spinosin, a C-glycoside flavonoid of Semen Ziziphi spinosae,

on pentobarbital-induced sleep may be related to postsynaptic 5-HT (1A) receptors," Phytomedicine, vol. 17, no. 6, pp. 404-409, 2010.

[9] J. Xie, L. Guo, G. Pang et al., "Modulation effect of Semen Ziziphi spinosae extracts on IL-1 $\beta$, IL-4, IL-6, IL-10, TNF- $\alpha$ and IFN- $\gamma$ in mouse serum," Natural Product Research, vol. 25, no. 4, pp. 464-467, 2011.

[10] X. F. Zhai, X. C. Xiao, Y. J. Lou et al., "The sedative and hypnotic effects on EEG in insomnia rats of raw and parched Semen Ziziphi spinosae," Pharmacology Clinical China Materials Medicine, vol. 31, pp. 94-97, 2015.

[11] L. E. Wang, Y. J. Bai, and X. R. Shi, "Spinosin, a C-glycoside flavonoid from Semen Zizhiphi spinozae, potentiated pentobarbital-induced sleep via the serotonergic system," Pharmacology Biochemistry and Behavior, vol. 90, no. 3, pp. 399-403, 2008.

[12] Z. Li, S. Ren, Z. R. Chang et al., "Content comparison of three components in Ziziphi spinosae Semen pieces," Chinese Journal of New Drugs, vol. 26, pp. 91-96, 2017.

[13] H. Y. Zhu, L. N. Zhang, S. Tang et al., "Determination of spinosin, jujubosides A and B in Ziziphi spinosae Semen from three different origins by HPLC," Chinese Journal of Pharmaceutical Analysis, vol. 35, pp. 2099-2104, 2015.

[14] F. X. Zhang, M. Li, L. R. Qiao et al., "Rapid characterization of Ziziphi spinosae Semen by UPLC/Qtof MS with novel informatics platform and its application in evaluation of two seeds from Ziziphus species," Journal of Pharmaceutical and Biomedical Analysis, vol. 122, pp. 59-80, 2016.

[15] A. Stavrianidi, E. Stekolshchikova, P. Anna, I. Rodin, and O. Shpigun, "Combination of HPLC-MS and QAMS as a new analytical approach for determination of saponins in ginseng containing products," Journal of Pharmaceutical and Biomedical Analysis, vol. 132, pp. 87-92, 2017.

[16] E. A. Turner, A. C. Stenson, and S. K. Yazdani, "HPLC-MS/ MS method for quantification of paclitaxel from keratin containing samples," Journal of Pharmaceutical and Biomedical Analysis, vol. 139, pp. 247-251, 2017.

[17] C. Gstottner, D. Klemm, M. Haberger et al., "Fast and automated characterization of antibody variants with 4D HPLC/ MS," Analytical Chemistry, vol. 90, no. 3, pp. 2119-2125, 2018.

[18] Y. Zhang, L. Qiao, M. Song et al., "Hplc-ESI-MS/MS analysis of the water-soluble extract from Ziziphi spinosae Semen and its ameliorating effect of learning and memory performance in mice," Pharmacognosy Magazine, vol. 10, no. 40, pp. 509-516, 2014.

[19] B. Yang, H. Yang, F. Chen, Y. Hua, and Y. Jiang, "Phytochemical analyses of Ziziphus jujuba Mill. var. spinosa seed by ultrahigh performance liquid chromatography-tandem mass spectrometry and gas chromatography-mass spectrometry," Analyst, vol. 138, pp. 6881-6888, 2013.

[20] B. Chen and A. G. Zhao, "The research status of Chinese medicine decoction and the major new types of Chinese medicine," World Chinese Medicine, vol. 9, pp. 396-399, 2014.

[21] Q. Y. Zhang, H. T. Yang, H. Shi et al., "Simultaneous quantification of nine constituents in Ziziphi spinosae Semen by HPLC-MS," China Traditional and Herbal Drugs, vol. 46, pp. 123-127, 2015. 

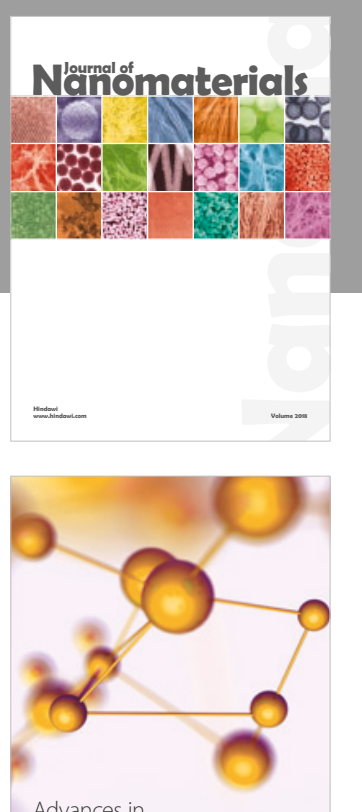

Physical Chemistry
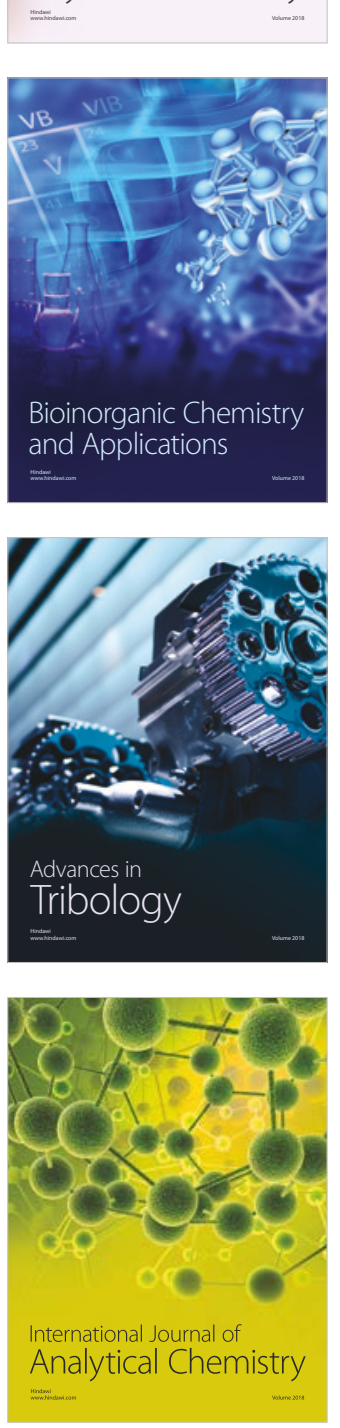

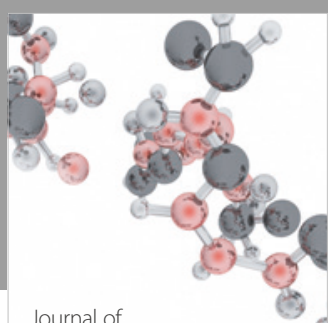

Analytical Methods

in Chemistry

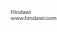

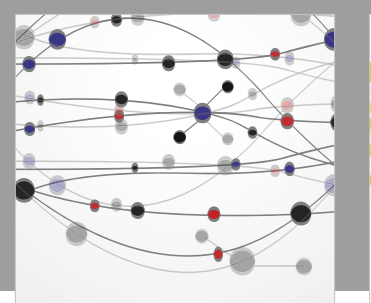

The Scientific World Journal

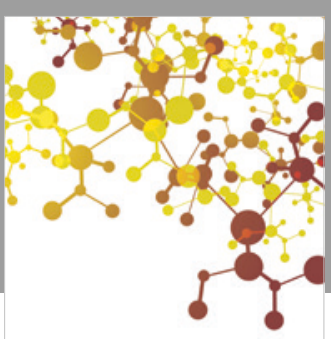

Journal of

Applied Chemistry
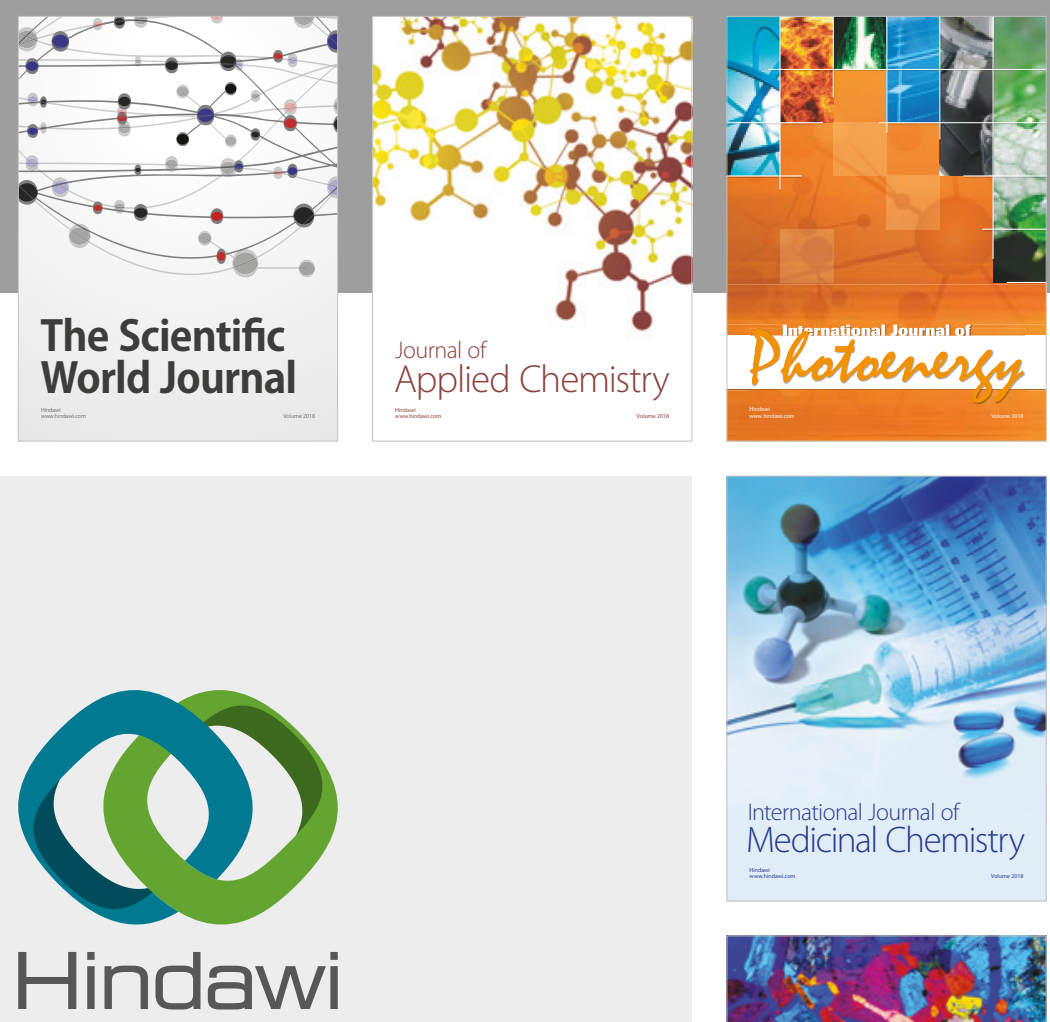

Submit your manuscripts at

www.hindawi.com
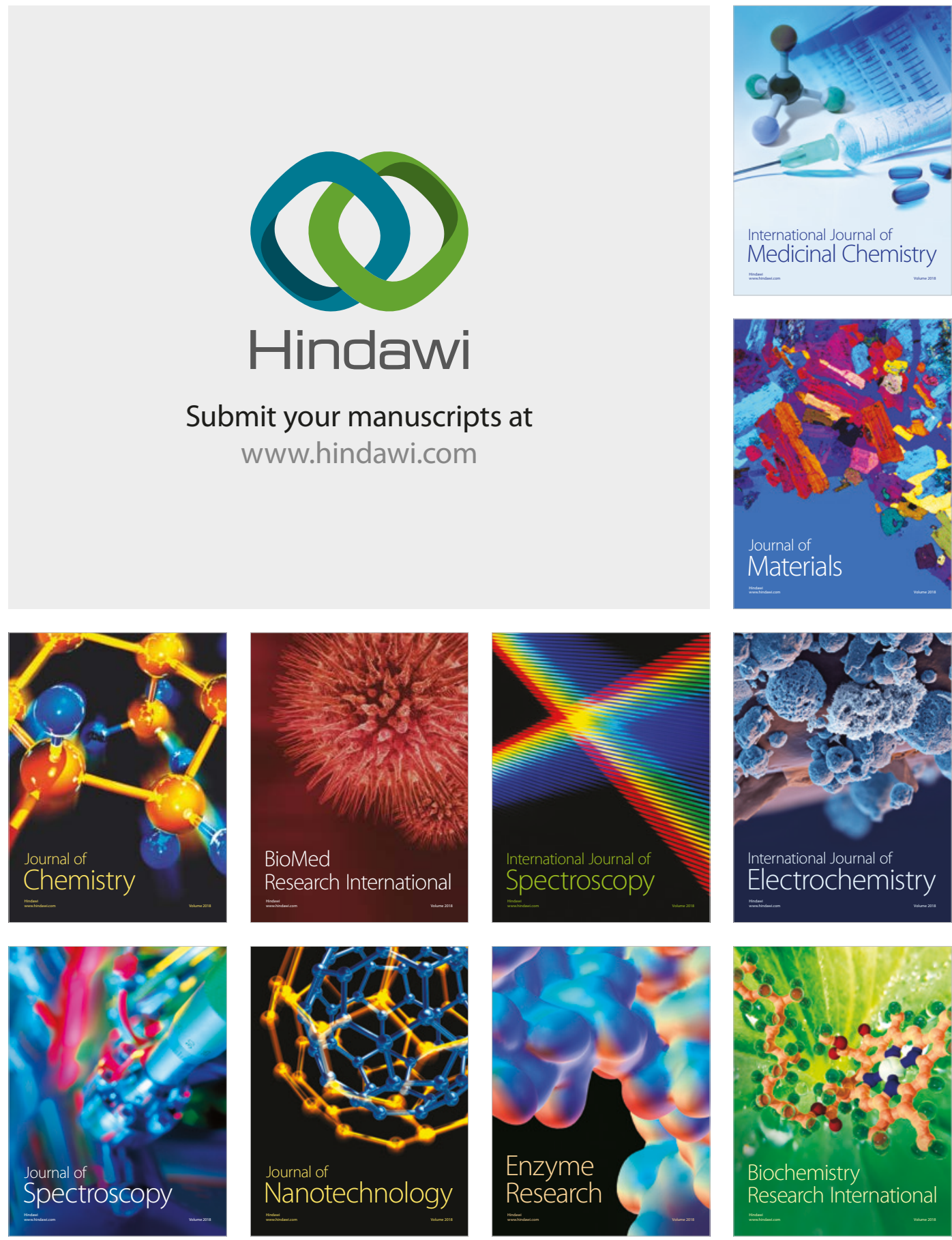
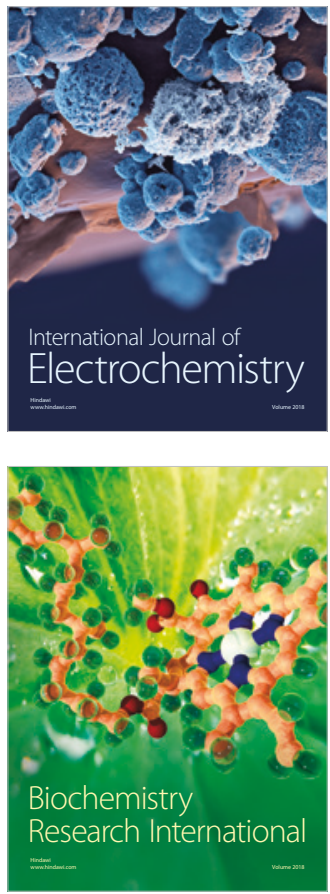